\title{
Computational Techniques for basic Affine Models of Portfolio Credit Risk
}

\author{
Andreas Eckner* \\ First version: August 2007 \\ Current version: August 2009 \\ Forthcoming in Journal of Computational Finance
}

\begin{abstract}
This paper presents computational techniques that make a certain class of fully dynamic intensity-based models for portfolio credit risk, along the lines of Duffie and Gârleanu (2001) and Mortensen (2006), just as computationally tractable as the Gaussian copula model. For this model, we improve the fit to tranche spreads by a factor of around three, by allowing for a more flexible correlation structure, and by accounting for market frictions due to bid-offer spreads. The resulting model can be used to hedge a wide range of risks in the credit market, such as the risk of changes in correlations, volatilities, or idiosyncratic default risk.
\end{abstract}

Keywords: credit risk, correlated default, structured credit derivatives, affine jump diffusion, intensity-based model, CDO pricing

${ }^{*}$ Department of Statistics, Stanford University. Comments are welcome at andreas@eckner.com. The source code of the implementation is available at http://www.eckner.com/research.html. I would like to thank Xiaowei Ding, Peter Feldhütter, Kay Giesecke, Tze Leung Lai, Allan Mortensen and George Papanicolaou for helpful comments and remarks, and especially Darrell Duffie for frequent discussions. I am grateful to Citi, Markit and Morgan Stanley for providing historical credit index and tranche spreads, and Markit for providing historical CDS spread data. 


\section{Introduction}

We present computational techniques that make a fully dynamic, doubly stochastic, intensity-based model ${ }^{1}$ for the joint behavior of corporate default times, along the lines of Duffie and Gârleanu (2001) and Mortensen (2006), just as tractable as the static Gaussian copula model ( $\mathrm{Li}(2000)$ ). Both model implementations have the recursive calculation of the conditional (on the common factor) portfolio loss distribution, using an algorithm due to Andersen, Sidenius, and Basu (2003), as the computational bottleneck. ${ }^{2}$ In our implementation of this intensity-based model, the "ASB"-algorithm accounts for $65 \%$ of the total computing time when pricing credit tranches. A modified version of the algorithm that avoids calculating the probability of extremely unlikely events, still accounts for $38 \%$ of the total computing time. Credit tranches can be priced in less than one second on a single-processor workstation, ${ }^{3}$ while calibration of the model for a single day takes only a few minutes, given the fitted parameters from the previous day.

A second emphasis of this paper is to improve upon the model fit in Mortensen (2006) by a factor of around three, by allowing for a more flexible correlation structure, and by accounting for market frictions due to bid-offer spreads. We also show that a rich class of recovery rate scenarios can be incorporated into the model in a computationally tractable manner. The resulting model can be used to hedge a wide range of risks in the credit market, such as the risk of changes in spread volatilities, or idiosyncratic default risk - effects that cannot be considered in the inherently static copula model.

A tractable dynamic model for portfolio credit risk should be of interest to a variety of researchers and practitioners. Although the Gaussian copula model is an industry standard, its theoretical foundations such as implied default and credit spread dynamics are often quite unrealistic. Tranches of CDOs usually cannot be priced consistently using a single correlation parameter, which has given rise to the base correlation framework, even though it does not guarantee arbitrage-free prices. For these reasons, various authors have considered extensions such as the Clayton, Student-t, double-t, or MarshallOlkin copulas. See Burtschell, Gregory, and Laurent (2005) for a comparative analysis. Nevertheless, current models of this type cannot capture the dynamics of credit spreads and are therefore unsuitable analyzing certain risk dimensions (such as mark-to-market risk and collateral agreements ${ }^{4}$ ) and for pricing securities whose payout depends on credit

\footnotetext{
${ }^{1}$ Standard references on intensity-based models, sometimes called reduced-form models, include Jarrow and Turnbull (1995), Lando (1998), and Duffie and Singleton (1999).

${ }^{2}$ Jackson, Kreinin, and Ma (2007) found this algorithm to be preferable to Fourier based convolution methods, as long as the portfolio contains only a couple of hundred issuers. From talking with practitioners we learned that the recursive Andersen-Sidenius-Basu step currently is indeed the preferred method for the one-factor Gaussian copula model.

${ }^{3}$ This computing time applies to a hybrid C/Matlab model implementation on a computer with $1.86 \mathrm{Ghz}$ Intel $\AA$ Celeron $\AA$ processor with $1 \mathrm{~GB}$ of RAM. A pure $\mathrm{C}$ implementation would likely be two to three times faster.

${ }^{4}$ Incorrect assessment of the later type of risk triggered a liquidity crisis at American In-
} 
spread dynamics (such as options on CDS indices). On the other hand, these features can be incorporated in a natural way into an intensity-based framework of credit risk. Due to the rapid growth of the market for credit derivatives over the past decade, intensity-based models of credit risk are currently an active area of research. See for example Duffie and Gârleanu (2001), Giesecke and Goldberg (2005), Errais, Giesecke, and Goldberg (2006), Joshi and Stacey (2006), Mortensen (2006), Papageorgiou and Sircar (2008), and Longstaff and Rajan (2008).

In our analysis we rely heavily on the doubly stochastic assumption for default intensities, so that correlation of default intensities is the only mechanism by which correlation of default times can arise. While computationally convenient, it is important to keep in mind that this framework rules out some important other channels of default correlation, such as unobservable risk-factors (frailty) and contagion. See, for example, Das, Duffie, Kapadia, and Saita (2007), Duffie, Eckner, Horel, and Saita (2009), and Ding, Giesecke, and Tomecek (2008). However, for alternative bottom-up specifications that relax the doubly-stochastic assumption, computational tractability may an issue. As opposed to a bottom up model as ours, where the primitives are the intensity processes of the individual firms, top-down models directly specify the portfolio loss process in terms of an intensity process. Such models more easily allow the relaxation of the doubly stochastic assumption while retaining computational tractability. However, additional non-trivial steps are required to calibrate the single names in the portfolio.

The rest of the paper is organized as follows. The remainder of this Section describes some of the most common credit derivatives and the data sources used for our analysis. Section 2 introduces the model for risk-neutral default times, while Section 3 presents various computational techniques. Section 4 present the pricing of credit derivatives in this setup and the model calibration algorithm. Section 5 examines the model fit at a fixed point in time and discusses hedging of credit tranches. Section 6 concludes and suggests possible model extensions.

\subsection{Credit Derivative Contracts}

A credit derivative is a contract whose payoff is linked to the creditworthiness of one or more obligations. This section summarizes the features of the three most common types for corporate credit risk.

Credit Default Swaps. By far the most common credit derivative is the credit default swap (CDS). It is an agreement between a protection buyer and a protection seller, whereby the buyer pays a periodic fee in return for a contingent payment by the seller upon a credit event, such as bankruptcy or "failure to pay", of a reference entity.

ternational Group in September 2008 with subsequent rescue by the Federal Reserve. See http://www.federalreserve.gov/newsevents/press/other/20080916a.htm for details. 
The contingent payment usually replicates the loss incurred by a creditor of the reference entity in the event of its default. See, for example, Duffie (1999).

Credit Indices. A credit index contract allows an investor to either buy or sell protection on a basket of reference entities, and therefore closely resembles a portfolio of CDS contracts. For example, the CDX.NA.IG (for CDS index, North America, Investment Grade) contract provides equally-weighted default protection on 125 North American investment-grade rated issuers. For a more detailed description see the 'Credit Derivatives Handbook' (2006) by Merrill Lynch.

Credit Tranches. A credit tranche allows an investor to gain a specified exposure to the credit risk of the underlying portfolio, while in return receiving periodic coupon payments. Losses due to credit events in the underlying portfolio are allocated first to the lowest tranche, known as the equity tranche, and then to successively prioritized tranches. The risk of a tranche is determined by the attachment point of the tranche, which defines the point at which losses in the underlying portfolio begin to reduce the tranche notional, and the detachment point, which defines the point at which losses in the underlying portfolio reduce the tranche notional to zero. The buyer of protection makes coupon payments on the notional amount of the remaining size of the tranche, which is the initial tranche size less losses due to defaults. This structure is illustrated in Table 1 for the CDX.NA.IG index.

\begin{tabular}{l|rrr}
\hline \hline Tranche & Attachment Point & Detachment Point & Quote Convention \\
\hline Equity & $0 \%$ & $3 \%$ & 500 bps running + upfront \\
Junior Mezzanine & $3 \%$ & $7 \%$ & all running \\
Mezzanine & $7 \%$ & $10 \%$ & all running \\
Senior Mezzanine & $10 \%$ & $15 \%$ & all running \\
Senior & $15 \%$ & $30 \%$ & all running \\
Super Senior & $30 \%$ & $100 \%$ & all running \\
\hline \hline
\end{tabular}

Table 1: Tranche structure of the CDX.NA.IG index, which has 125 equally weighted North-American investment-grade issuers in the underlying portfolio.

The coupon payments for these three contracts are typically made quarterly, on the 20th of March, June, September, and December, unless this date is a holiday, in which case the payment is made on the next business day. If a contract is entered in between two such dates, the buyer of protection receives from the seller of protection the accrued premium since the last coupon date. 


\subsection{Data Sources}

Citi and Morgan Stanley provided 5, 7, and 10-year CDX.NA.IG index and tranche bidand ask-spreads. Markit provided 5, 7, and 10-year CDX.NA.IG tranche mid-market spreads, and 1, 5, 7, and 10-year CDS mid-market spreads for the CDX.NA.IG members.

We used 3-month, 6-month, 9-month, 1-year, 2-year, ..., 10-year US LIBOR swap rates to estimate the discount function $B_{t}(T)$ at each point in time $t$. Specifically, for these standard maturities we used swap rates from the Bloomberg system, while for non-standard maturities we used cubic-spline interpolation of implied forward rates to determine the spot rate. Swap rates are widely regarded as the best measure of funding costs for banks - the biggest participant in the market for synthetic credit derivatives over various time horizons.

\section{Model Setup}

This section describes a model for the joint distribution of various obligor default times under a risk-neutral probability measure. To this end, we fix a filtered probability space $\left(\Omega, \mathcal{F},\left(\mathcal{F}_{t}\right), \mathbb{P}\right)$ satisfying the usual conditions. ${ }^{5}$ Up to purely technical conditions, ${ }^{6}$ the absence of arbitrage implies the existence of an equivalent martingale measure $\mathbb{Q}$, such that the price at time $t$ of a security paying an amount $Z$ at a stopping time $\tau>t$ is given by

$$
V_{t}=E_{t}^{\mathbb{Q}}\left(e^{-\int_{t}^{\tau} r_{s} d s} Z\right)
$$

where $r$ is the short-term interest rate and $E_{t}^{\mathbb{Q}}$ denotes expectation under $\mathbb{Q}$, conditional on all available information up to time $t$.

Under the equivalent martingale measure $\mathbb{Q}$, for each individual firm $i$, a default time $\tau_{i}$ is modeled using Cox processes, also known as doubly stochastic Poisson processes. See for example Lando (1998) and Duffie and Singleton (2003). Specifically, the default intensity of obligor $i$ is a non-negative real-valued progressively measurable stochastic process, which will be defined below. Conditional on the intensity path $\left\{\lambda_{i t}: t \geq 0\right\}$, the default time $\tau_{i}$ is taken to be the first jump time of an inhomogeneous Poisson process with intensity $\lambda_{i}$. In particular, the default times of any set of firms are conditionally independent given the intensity paths, so that correlation of default intensities is the only mechanism by which correlation of default times can arise.

For $t>s$, risk-neutral survival probabilities can be calculated via

$$
\mathbb{Q}\left(\tau_{i}>t \mid \mathcal{F}_{s}\right)=E_{s}^{\mathbb{Q}}\left(\mathbb{Q}\left(\tau_{i}>t \mid\left\{\lambda_{i t}: t \geq 0\right\} \cup \mathcal{F}_{s}\right)\right)=\mathbf{1}_{\left\{\tau_{i}>s\right\}} E_{s}^{\mathbb{Q}}\left(e^{-\int_{s}^{t} \lambda_{i u} d u}\right),
$$

\footnotetext{
${ }^{5}$ For a precise mathematical definition not offered here, see Karatzas and Shreve (2004), and Protter (2005).

${ }^{6}$ See Harrison and Kreps (1979), Harrison and Pliska (1981), and Delbaen and Schachermayer (1999).
} 
where the expectation is taken over the distribution of possible intensity paths. The large and flexible class of affine processes allows one to calculate (1) either explicitly or numerically quite efficiently. See Duffie, Pan, and Singleton (2000).

Due to their computational tractability, we use basic Affine Jump Diffusions (AJD) as the building block for the default intensity model. Specifically, we call a stochastic process $Z$ a basic AJD under $\mathbb{Q}$ if

$$
d Z_{t}=\kappa\left(\theta-Z_{t}\right) d t+\sigma \sqrt{Z_{t}} d B_{t}+d J_{t}, \quad Z_{0} \geq 0
$$

where under $\mathbb{Q},\left(B_{t}\right)_{t \geq 0}$ is a standard Brownian motion, and $\left(J_{t}\right)_{t \geq 0}$ is an independent compound Poisson process with constant jump intensity $l$ and exponentially distributed jumps with mean $\mu$. For the process to be well defined, we require that $\kappa \theta \geq 0$ and $\mu \geq 0$. Note that (2) is a special case of more general affine processes, see for example Duffie, Filipović, and Schachermayer (2003).

Basic AJDs are especially attractive for modeling default times, since both the moment generating function

$$
m(q)=E^{\mathbb{Q}}\left(e^{q \int_{0}^{t} Z_{s} d s}\right), \quad q \in \mathbb{R},
$$

and the characteristic function

$$
\varphi(u)=E^{\mathbb{Q}}\left(e^{i u \int_{0}^{t} Z_{s} d s}\right), \quad u \in \mathbb{R},
$$

are known in closed-from, see Appendix A for details. In particular, if $Z$ is the default intensity of a certain obligor, setting $q=-1$ in (3) allows one to explicitly calculate the obligor's survival probability (1). In addition, the characteristic function (4) allows one to calculate the density of an integrated basic AJD

$$
\widetilde{Z}_{t} \equiv \int_{0}^{t} Z_{s} d s
$$

by Fourier inversion, as discussed in Section 3.2 below.

\subsection{Risk-Neutral Default Intensities}

We now make precise the multivariate model of default times. The risk-neutral default intensity of obligor $i$ is

$$
\lambda_{i t}=X_{i t}+a_{i} Y_{t}
$$

with idiosyncratic component $X_{i}$ and systematic component $Y$. As in Duffie and Gârleanu (2001) and Mortensen (2006), under $\mathbb{Q}, X_{1}, \ldots, X_{m}$ and $Y$ are independent basic AJDs, with

$$
\begin{aligned}
d X_{i t} & =\kappa_{i}\left(\theta_{i}-X_{i t}\right) d t+\sigma_{i} \sqrt{X_{i t}} d B_{t}^{(i)}+d J_{t}^{(i)} \\
d Y_{t} & =\kappa_{Y}\left(\theta_{Y}-Y_{t}\right) d t+\sigma_{Y} \sqrt{Y_{t}} d B_{t}^{(Y)}+d J_{t}^{(Y)} .
\end{aligned}
$$


Here, $J^{(Y)}$ and $J^{(i)}$ have jump intensities $l_{Y}$ and $l_{i}$, and jump size means $\mu_{Y}$ and $\mu_{i}$, respectively. Hence, jumps can either be firm-specific or market-wide. Duffie and Gârleanu (2001) and Mortensen (2006) found the latter type of jumps in default intensities to be crucial for explaining the spreads of senior CDO tranches, which are heavily exposed to tail risk events. Schneider, Sögner, and Veža (2009) examined the time series of 282 credit default swap spreads and found evidence for mainly positive jumps in default intensities.

\subsubsection{Parameter Restrictions}

This section discusses restrictions on the parameters in (6) and (7) that (i) make the model identifiable and (ii) reduce, for parsimony, the number of free parameters. We also point out the differences between our model setup and those of Duffie and Gârleanu (2001) and Mortensen (2006). For a discussion about the suitability of such restrictions see Feldhütter (2007).

Model Identifiability. The restriction

$$
\frac{1}{m} \sum_{i=1}^{m} a_{i}=1
$$

is imposed to ensure identifiability of the model. ${ }^{7}$

Parsimony. Our model specification is relatively general with $5 m+5$ default intensity parameters and $m+1$ initial factor values. Since we are especially interested in the economic interpretation of the parameters, we favor a parsimonious model which is nevertheless flexible enough to closely fit tranches spreads. First, we take the common factor loading $a_{i}$ of each obligor $i$ to be equal to the obligor's 5-year CDS spread divided by the average 5 -year CDS spread of the current credit index members, that is

$$
a_{i}=\frac{c_{i, t, M}^{\mathrm{cds}}}{\operatorname{Avg}_{i}\left(c_{i, t, M}^{\mathrm{cds}}\right)},
$$

\footnotetext{
${ }^{7}$ If all factor loadings $a_{i}$ are replaced by $c a_{i}$ for some positive constant $c$, then replacing the parameters $\left(Y_{0}, \kappa_{Y}, \theta_{Y}, \sigma_{Y}, l_{Y}, \mu_{Y}\right)$ with $\left(Y_{0} / c, \kappa_{Y}, \theta_{Y} / c, \sigma_{Y} / \sqrt{c}, l_{Y}, \mu_{Y} / c\right)$ leaves the dynamics of $a_{i} Y$ (and therefore also the joint dynamics of $\lambda_{i}$ ) unchanged.
} 
where $c_{i, t, M}^{\text {cds }}$ denotes the 5 -year CDS spread at time $t$ for the $i$ th reference entity. Moreover, we impose the parameter constraints

$$
\begin{aligned}
\kappa_{i} & =\kappa_{Y} \equiv \kappa, \\
\sigma_{i} & =\sqrt{a_{i}} \sigma_{Y} \equiv \sqrt{a_{i}} \sigma, \\
\mu_{i} & =a_{i} \mu_{Y} \equiv a_{i} \mu, \\
\omega_{1} & =\frac{l_{Y}}{l_{i}+l_{Y}} \\
\omega_{2} & =\frac{a_{i} \theta_{Y}}{a_{i} \theta_{Y}+\theta_{i}},
\end{aligned}
$$

which reduces the number of free parameters to just seven. Feldhütter (2007) examines to what extent (8)-(13) are empirically supported by CDS data for firms in the CDX.NA.IG index, and finds these assumptions in general to be fairly reasonable.

The constraints (8)-(13) imply that $\lambda_{i}$ is a basic AJD, which is not generally the case for the sum of two basic AJDs, see Duffie and Gârleanu (2001), Proposition 1. Specifically,

$$
d \lambda_{i t}=\kappa\left(\left(\theta_{i}+a_{i} \theta_{Y}\right)-\lambda_{i t}\right) d t+\sqrt{a_{i}} \sigma \sqrt{\lambda_{i t}} d \widetilde{B}_{t}^{(i)}+d \widetilde{J}_{t}^{(i)},
$$

or in short-hand

$$
\begin{aligned}
\lambda_{i} & =\operatorname{bAJD}\left(\lambda_{i, 0}, \kappa, \theta_{i}+a_{i} \theta_{Y}, \sqrt{a_{i}} \sigma_{Y}, l_{i}+l_{Y}, \mu_{i}\right)= \\
& =\operatorname{bAJD}\left(\lambda_{i, 0}, \kappa, \widetilde{\theta}_{i}, \widetilde{\sigma}_{i}, \widetilde{l}, \mu_{i}\right),
\end{aligned}
$$

where $\widetilde{\theta}_{i}=\theta_{i}+a_{i} \theta_{Y}$ and $\widetilde{l}=l_{i}+l_{Y}$.

It is easy to show that $\theta_{Y}=\omega_{2} \operatorname{Avg}\left(\widetilde{\theta}_{i}\right) \equiv \omega_{2} \widetilde{\theta}_{\text {Avg }}$ and that $\widetilde{\theta}_{i}=a_{i} \widetilde{\theta}_{\text {Avg }}$ for each $i$, so that we can characterize the joint risk-neutral model of default times with the seven parameters

$$
\Theta=\left\{\kappa, \tilde{\theta}_{\mathrm{Avg}}, \sigma, \widetilde{l}, \mu, \omega_{1}, \omega_{2}\right\},
$$

and the $m+1$ initial factor values. Even though the above constraints greatly reduce the number of free parameters, a model without these constraints would be just as computationally tractable, since the computational techniques described below do not make use of (8)-(13).

Remark 1 The model setup in this section is slightly more general than that of Mortensen (2006), which in turn is a generalization of Duffie and Gârleanu (2001), who examined a homogenous portfolio so that in particular all factor loadings $a_{i}$ are equal to one. Mortensen (2006) imposed

$$
\omega_{1}=\omega_{2}=\frac{Y_{0}}{Y_{0}+\operatorname{Avg}\left(X_{i 0}\right)},
$$

so that the jump-correlation structure and mean-reversion levels are determined by the initial values of the $m+1$ factors. 


\section{Computational Techniques}

This section describes computational techniques for pricing credit derivatives in the basic AJD framework of Section 2. Apart from Section 3.1 and 3.2, the techniques are applicable to other credit derivative pricing models as well. The modified AndersenSidenius-Basu algorithm, for example, allows one to compute the conditional portfolio loss distribution for any factor model, no matter whether dynamic or static, where default times are conditionally independent given the common factors. Similarly, the model for stochastic and serially correlated recovery rates applies to any model where recovery rates are independent of default times.

\subsection{Survival Probabilities}

In the affine two-factor model of Section 2.1,

$$
\mathbb{Q}\left(\tau_{i}>t \mid \mathcal{F}_{s}\right)=\mathbf{1}_{\left\{\tau_{i}>s\right\}} E_{s}^{\mathbb{Q}}\left[e^{-\int_{s}^{t} X_{i, u} d u}\right] E_{s}^{\mathbb{Q}}\left[e^{-a_{i} \int_{s}^{t} Y_{u} d u}\right] .
$$

The expectations on the right-hand side can be calculated explicitly by evaluating the moment generating function (3) at $q=-1$.

\subsection{Common Factor Distribution}

Mortensen (2006) derived a system of ODEs, whose solution yields the characteristic function (4). Appendix A.2 shows that this characteristic function is actually known in closed-form. The distribution of the integrated common factor

$$
\widetilde{Y}_{s, t} \equiv \int_{s}^{t} Y_{u} d u
$$

can therefore be efficiently calculated by Fourier inversion, which was done by carrying out the following steps:

1. Evaluate the characteristic function of $\widetilde{Y}_{s, t}$ on an unequally spaced grid of length 1024 with mesh size smallest for grid points close to 0, for example by using an equally-spaced grid on a logarithmic scale.

2. Fit a complex-valued cubic spline to the output from step 1, and evaluate the cubic spline on an equally spaced grid with $2^{18}$ points.

3. Apply the Fast Fourier Transform (FFT) to the output from step 2 to obtain the density of $\widetilde{Y}_{s, t}$ evaluated on an equally-spaced grid.

See Cerny (2004) for a general introduction to the FFT, and Carr and Madan (1999), Section 4 for details regarding the spacing of input and output points. 


\subsection{Conditional Distribution of Defaults}

By construction, conditional on the integrated common factor $\widetilde{Y}_{s, t}$, defaults in the time interval $(s, t]$ occur independently. At time $s$, the conditional distribution $P_{s, t}$ of the number of defaults in the portfolio up to time $t$ can therefore be computed using the recursive algorithm of Andersen, Sidenius, and Basu (2003) (ASB in the following). Specifically, let

$$
q_{i}\left(\widetilde{Y}_{s, t}\right)=\mathbb{Q}_{s}\left(s<\tau_{i} \leq t \mid \widetilde{Y}_{s, t}\right)=\mathbf{1}_{\left\{\tau_{i}>s\right\}}\left(1-E_{s}^{\mathbb{Q}}\left[e^{-\int_{s}^{t} X_{i, u} d u}\right] e^{-a_{i} \widetilde{Y}_{s, t}}\right)
$$

denote the conditional default probability of the $i$ th issuer, and $P_{s, t}^{(n)}\left(k \mid \widetilde{Y}_{s, t}\right)$ denote the conditional probability that $k$ of the first $n$ credits in the portfolio default between times $s$ and $t$. The following recursive updating scheme allows one to calculate the distribution of the number of defaults in the portfolio conditional on $\widetilde{Y}_{s, t}$ :

$$
\begin{aligned}
P_{s, t}^{(0)}\left(k \mid \widetilde{Y}_{s, t}\right) & =\mathbf{1}_{\{k=0\},} \\
P_{s, t}^{(n+1)}\left(k \mid \widetilde{Y}_{s, t}\right) & =q_{n+1}\left(\widetilde{Y}_{s, t}\right) P_{s, t}^{(n)}\left(k-1 \mid \widetilde{Y}_{s, t}\right)+\left(1-q_{n+1}\left(\widetilde{Y}_{s, t}\right)\right) P_{s, t}^{(n)}\left(k \mid \widetilde{Y}_{s, t}\right),
\end{aligned}
$$

for $0 \leq k \leq n$ and $0 \leq n<m$.

Modified ASB-Algorithm The algorithm (16) can be modified so as to considerably increase its speed. Specifically, we restrict the ASB-algorithm to values of $k$, such that $P_{s, t}\left(k \mid \tilde{Y}_{s, t}\right)>\varepsilon$ for some small number $\varepsilon$, for example $10^{-10}$. Otherwise, the algorithm spends a large amount of time computing the probability of events that are extremely unlikely to occur, and therefore have a negligible impact on credit tranche spreads. ${ }^{8}$

To this end, for a given value of the integrated common factor $\widetilde{Y}_{s, t}$, let

$$
d\left(\widetilde{Y}_{s, t}\right)=d\left(\widetilde{Y}_{s, t}, s, t\right)=\sum_{i=1}^{m} q_{i}\left(\widetilde{Y}_{s, t}\right)
$$

denote the conditional (on the integrated common factor) expected number of defaults in the portfolio during the time interval $(s, t]$. Under certain conditions, the Poisson approximation (see for example Durrett (2005), Theorem 6.1) implies that $P_{s, t}\left(k \mid \widetilde{Y}_{s, t}\right)$ is close to a Poisson distribution with parameter $d\left(\widetilde{Y}_{s, t}\right)$ so that

$$
P_{s, t}\left(k \mid \widetilde{Y}_{s, t}\right) \approx \frac{d\left(\widetilde{Y}_{s, t}\right)^{k} e^{-d\left(\widetilde{Y}_{s, t}\right) k}}{k !} .
$$

\footnotetext{
${ }^{8} \mathrm{~A}$ trivial but significant speed-up can also be achieved by replacing $\exp \left(-a_{i} \widetilde{Y}_{s, t}\right)$ in (15) with its Taylor series approximation for small values of $a_{i} \widetilde{Y}_{s, t}$.
} 
Hence, we restrict the ASB-algorithm in (16) to values $k \leq K(\varepsilon)$, where

$$
K(\varepsilon)=\min \left\{K: \frac{d\left(\widetilde{Y}_{s, t}\right)^{l} e^{-d\left(\widetilde{Y}_{s, t}\right) l}}{l !}<\varepsilon \text { for } l \geq K\right\},
$$

which leads to significant computational savings, since the conditional expected number of defaults $d\left(\widetilde{Y}_{s, t}\right)$ is usually quite small, so that $K(\varepsilon)$ is small too. ${ }^{9}$

\subsection{Unconditional Distribution of Defaults}

Recall that the output of the Fourier inversion steps is the density of $\widetilde{Y}_{s, t}$ evaluated on an equally spaced grid $\left(\widetilde{y}_{i}=i \Delta \widetilde{y}: 0 \leq i<N\right)$, where $\Delta \widetilde{y}$ is the spacing of the grid, and $N$ the number of points used in the FFT. The unconditional probability $P_{s, t}(k)$ of $k$ defaults in the portfolio can therefore be obtained by integration of $P_{s, t}\left(k \mid \widetilde{Y}_{s, t}\right)$ over the distribution of $\widetilde{Y}_{s, t}$ :

$$
\begin{aligned}
P_{s, t}(k) & =\int P_{s, t}\left(k \mid \widetilde{Y}_{s, t}\right) d \mathbb{Q}\left(\widetilde{Y}_{s, t}\right) \approx \\
& \approx \sum_{i=1}^{N} P_{s, t}\left(k \mid \widetilde{y}_{i}\right) f_{\widetilde{Y}_{s, t}}\left(\widetilde{y}_{i}\right) \Delta \widetilde{y} \equiv \widehat{P}_{s, t}(k)
\end{aligned}
$$

Since the integrand in (18) is quite costly to evaluate, this computation is extremely time-consuming for large values of $N$. Nevertheless, this calculation can be done quite efficiently by exploiting the smoothness of the probability $P_{s, t}\left(k \mid \widetilde{Y}_{s, t}\right)$ in $\widetilde{Y}_{s, t}$.

To this end, let $F_{\widetilde{Y}_{s, t}}$ denote the cumulative distribution function of $\widetilde{Y}_{s, t}$. For some number $N_{2} \ll N$, calculate the quantiles

$$
\gamma_{i}=F_{\widetilde{Y}_{s, t}}^{-1}\left(\frac{i}{N_{2}}\right), \quad 0 \leq i \leq N_{2} .
$$

With this notation, (18) can be written as a sum of integrals

$$
P_{s, t}(k)=\sum_{i=1}^{N_{2}} \int_{\gamma_{i-1}}^{\gamma_{i}} P_{s, t}\left(k \mid \tilde{Y}_{t}\right) d \mathbb{Q}_{s}\left(\tilde{Y}_{s, t}\right) .
$$

The integrals in (19) can be evaluated, for example, by Gauss-Legendre integration, and we denote the resulting approximation of the distribution $P_{s, t}$ by $\widetilde{P}_{s, t}$. ${ }^{10}$

\footnotetext{
${ }^{9}$ We found using a boundary value of $K=8+d\left(\widetilde{Y}_{s, t}\right)+8 \sqrt{d\left(\widetilde{Y}_{s, t}\right)}$ to work just as well, but faster to calculate than (17). The functional form of this expression is motivated by that fact that the mean and standard deviation of a Poisson random variable with parameter $\lambda$ are equal to $\lambda$ and $\sqrt{\lambda}$, respectively.

${ }^{10}$ Alternatively, one could use an adaptive quadrature method. However, since the distribution of $\widetilde{Y}_{s, t}$ tends to be highly skewed with very narrow peak and since the integrand in (18) is costly to evaluate, we found it advantageous to aid the numerical quadrature procedure by breaking down the integration range into sub-intervals based on the quantiles of the distribution of $\widetilde{Y}_{s, t}$.
} 
We found using $N_{2}=250$ and Gauss-Legendre integration with five support points to be very fast and accurate for calculating the unconditional distribution $P_{s, t}$ of the number of defaults. For the fitted models of Section 5.1, the approximation error from the numerical integration was found to satisfy

$$
\left\|\widetilde{P}_{s, t}-\widehat{P}_{s, t}\right\|_{T V}=\frac{1}{2} \sum_{k=0}^{m}\left|\widetilde{P}_{s, t}(k)-\widehat{P}_{s, t}(k)\right|<10^{-7}
$$

for a time horizon $t-s$ equal to five years, where $\|\cdot\|_{T V}$ denotes the total variation norm. ${ }^{11}$

\subsection{Multiple Horizons}

This sections discusses an interpolation method for efficiently calculating the unconditional distribution of the number of defaults $P_{s, t}$ for multiple time horizons $t$. Since the loss distribution $P_{s, t}$ varies smoothly in $t$, it is only necessary to calculate this distribution on a sparse grid of time horizons $\mathcal{T}=\left\{t_{0}, t_{1}, \ldots, t_{l}\right\}$. As hinted by Mortensen (2006), interpolation techniques can then be used to evaluate $P_{s, t}$ at non-grid points.

To this end, assume $P_{s, t}$ is already known for $t \in \mathcal{T}$ and that we want to compute $P_{s, t}$ for a time horizon $t$ with $t \notin \mathcal{T}$. For each $k$ with $0 \leq k \leq m$, let $w_{t}(k)$ denote the cubic spline interpolation of the data $\left\{\left(t_{j}, P_{s, t_{j}}(k)\right): 0 \leq j \leq l\right\}$ evaluated at $t$. Then

$$
\widehat{P}_{s, t}(k)=\frac{w_{t}(k)}{\sum_{n=0}^{m} w_{t}(n)}, \quad 0 \leq k \leq m,
$$

can serve as an estimate of the distribution $P_{s, t}$ of the number of defaults in the time interval $(s, t]$.

For the fitted model of Section 5.1, an analysis of the approximation error as a function of the grid spacing in $\mathcal{T}$ showed that the approximation (20) is extremely accurate up to a yearly spacing. Even a coarse two-year spacing resulted in a relative pricing error of less than $0.2 \%$ for each single tranche. To summarize, interpolating the distribution of the number of defaults $P_{s, t}$ over $t$ can speed up the pricing of credit tranches by a factor of at least four.

\subsection{Recovery Rates and Portfolio Loss Distribution}

A large portion of tranche pricing models in the literature, such as the Gaussian Copula model, implicitly assume deterministic recovery rates. However, a couple of empirical features of recovery rates should be considered:

\footnotetext{
${ }^{11}$ For the CDX tranches on this particular date, the approximation error resulted in a relative pricing error of less than $0.05 \%$ for each single tranche.
} 
1. Stochastic recovery rates: Moody's (2000) reports a large cross-sectional dispersion of defaulted debt recovery for senior unsecured bonds (often taken as the reference entities for credit default swaps) of US corporations between 1970 and 1998. The 25 th, 50 th and 75 th percentile of recovery rates were roughly $30 \%, 50 \%$ and $65 \%$, respectively. For 1989 to 1995, Altman and Kishore (1996) found recoveries for senior unsecured debt were on average $47 \%$ with a sample standard deviation of $27 \%$.

2. Correlation of recovery rates with macroeconomic conditions: Moody's (2000), and Altman, Bray, Resti, and Sironi (2005) find that recovery rates behave countercyclical, that is, recovery rates tend to be low when corporate default rates are high, and vice versa. In particular, recovery rates are positively serially correlated.

The remainder of this section shows how to incorporate stochastic and serially correlated recovery rates into the basic AJD framework of Section 2.1. To retain computational tractability, recovery rates are however still assumed to be independent of macroeconomic conditions, so that property one is fully, and property two partially incorporated into the model. To this end, let

$$
L_{s, t}(x)=\mathbb{Q}_{s}\left(\frac{1}{m} \sum_{i=1}^{m}\left(1-R_{i}\right) \mathbf{1}_{\left\{s<\tau_{i} \leq t\right\}} \leq x\right)
$$

denote the cumulative fractional portfolio loss distribution between times $s$ and $t$, where $x \in[0,1]$. Here $R_{i}$ denotes the recovery rate of the $i$ th firm, whose probabilistic properties will be defined shortly.

If, under $\mathbb{Q}$, recovery rates and default times are independent, then (21) can be written as

$$
L_{s, t}(x)=\sum_{k=0}^{m} P_{s, t}(k) G_{k}(x),
$$

where for each $k, G_{k}$ is the cumulative portfolio loss distribution conditional on $k$ defaults, which does not depend on the time points $s$ and $t$. Thus, one can incorporate a rich class of recovery rate scenarios, not only into the basic AJD framework of Section 2.1 , but into any model of portfolio credit risk that allows for the decomposition (22).

Example 1 The case of deterministic recovery rates equal to 40\%, a common industry 
practice for senior unsecured corporate debt, ${ }^{12}$ corresponds to the choice

$$
G_{k}(x)=1_{\left\{\frac{k}{m}(1-0.4) \leq x\right\}}
$$

Example 2 Stochastic recovery rates can be incorporated, for example, by fitting $G_{1}$ to historical data and taking

$$
G_{k}(x)=\left(G_{1} * G_{k-1}\right)(x)
$$

for $k \geq 2$, where '*' denotes the convolution operator for random variables. Section 5.1 examines the model fit for the particularly simple choice

$$
G_{1}(x)=\frac{2}{3} \mathbf{1}_{\{x=0.45\}}+\frac{1}{3} \mathbf{1}_{\{x=0.9\}},
$$

which matches the first two moments of historical recovery rates.

Appendix $\mathrm{C}$ discusses a choice for the conditional loss distributions $G_{k}$ that corresponds to stochastic and serially correlated recovery rates.

\section{Pricing and Model Calibration}

This section discusses the pricing of various credit derivatives and the model calibration to market prices.

\subsection{Pricing}

For the purpose of pricing credit risky securities, we adopt the widely used assumption:

Assumption 1 Under the risk-neutral probability measure $\mathbb{Q}$,

(i) Default intensities and interest rates are independent.

(ii) Recovery rates are independent of default intensities.

\footnotetext{
${ }^{12}$ As elaborated by Duffie and Singleton (1997), and Duffie and Singleton (1999) it is in general difficult to separately identify risk-neutral recovery rates and default intensities. Even in cases in which one has multi-horizon data available, as in Pan and Singleton (2008), recovery rate estimates seem to be sensitive to the chosen model. Even though Pan and Singleton (2008) focus on sovereign CDS contracts, they show that this assumption is quite innocuous as long as the unknown true expected recovery rate is not close to $100 \%$.
} 
The first assumption has been found to be fairly innocuous, for example, Brigo and Alfonsi (2004) find no significant correlation between default intensities and interest rates, although Feldhütter and Lando (2004) and Driessen (2005) find a slightly positive correlation. However, in light of the 2007-2008 credit crunch, this conclusion might have to be revised. Nevertheless, for CDOs during this credit crisis, interest rate risk has been dwarfed by credit spread and default risk. Regarding the second assumption, Altman, Bray, Resti, and Sironi (2005) and Moody's (2000) find that recovery rates tend to behave counter-cyclical, at least under the physical probability measure.

The general procedure for pricing credit derivatives is setting the value of the fixed leg (the market value of the payments made by the buyer of protection) equal to the value of the protection leg (the market value of the payments made by the seller of protection) and to solve for the fair credit spread.

\subsubsection{CDS and Credit Index Pricing}

It is well known (see Appendix B.1 for details) that under Assumption 1 it is only necessary to calculate the conditional survival probabilities (14) for the set of future coupon payment dates $\left\{t_{l}: 1 \leq l \leq n\right\}$, in order to price a CDS contract. Similarly, knowledge of these survival probabilities for all companies in a portfolio is sufficient for pricing the corresponding credit index contract. Hence, in the basic AJD framework of Section 2.1, model-implied CDS and credit index spreads can be calculated explicitly.

\subsubsection{Tranche Pricing}

For a given point in time $t$, let

$$
N_{j, t}^{\mathrm{tr}}=\left(\bar{A}_{j}-\underline{A}_{j}\right)-\left[\max \left(L_{s, t}-\underline{A}_{j}, 0\right)-\max \left(L_{s, t}-\bar{A}_{j}, 0\right)\right]
$$

denote the remaining notional size of tranche $j$, where $\underline{A}_{j}$ and $\bar{A}_{j}$ denote the tranche attachment and detachment point, respectively. As shown in Appendix B.3, calculating the market value of the fixed and protection leg of a tranche $j$ requires calculating the expected tranche size $E_{s}^{\mathbb{Q}}\left(N_{j, t_{l}}^{\mathrm{tr}}\right)$ for the set of future coupon payments dates, $\left\{t_{l}: 1 \leq l \leq n\right\}$. We therefore never need to calculate the portfolio loss distribution (22), but can directly compute the expected tranche size as

$$
E_{s}^{\mathbb{Q}}\left(N_{j, t_{l}}^{\mathrm{tr}}\right)=N\left[\left(\bar{A}_{j}-\underline{A}_{j}\right)-\sum_{k=0}^{m} P_{s, t_{l}}(k) I_{j}(k)\right],
$$

where

$$
I_{j}(k)=\int_{\underline{A}_{j}}^{1} \min \left(x-\underline{A}_{j}, \bar{A}_{j}-\underline{A}_{j}\right) d G_{k}(x)
$$

is the risk-neutral expected loss for tranche $j$ conditional on $k$ defaults. The integrals in (25) need to be computed only once, and can then be stored for later use. 


\subsection{Computing Times}

In summary, in the basic AJD framework of Section 2.1, model-implied CDS, credit index, and credit tranche spreads can be calculated explicitly, or at least quite efficiently, using various computational techniques. Table 2 lists the computing times for some commonly used operations. In particular, it shows that the modified ASB step takes up about $38 \%$ of the total computing time when pricing credit tranches, while the original version of the ASB algorithm would have accounted for more than $65 \%$. The overall computational tractability of the basic AJD model is therefore on the same order of magnitude as that of the static Gaussian copula model, which, in its currently preferred implementation, also relies on the recursive ASB step (16).

\begin{tabular}{l|r|r}
\hline \hline Task & Environment & \% of Time \\
\hline Evaluating the characteristic function (4) & $\mathrm{C}$ & $7.5 \%$ \\
Cubic spline interpolation of characteristic function & $\mathrm{C} \&$ Matlab & $24.1 \%$ \\
FFT & Matlab & $16.5 \%$ \\
Modified ASB-algorithm & $\mathrm{C}$ & $38.3 \%$ \\
Other & Matlab & $13.5 \%$ \\
\hline \hline
\end{tabular}

Table 2: Percentage of time spent on various operations when calculating tranche spreads in the basic AJD framework of Section 2. This breakdown of computing times applies to a hybrid C/Matlab model implementation on a computer with $1.86 \mathrm{Ghz}$ Intel $\AA$ Celeron $\AA$ processor with $1 \mathrm{~GB}$ of RAM. Computing times exclude internal Matlab overhead, which was less than $25 \%$.

\subsection{Market Frictions}

When pricing credit derivatives one must account for various market frictions, as well as for certain trading conventions in this market.

First, credit indices and credit tranches recognize only bankruptcy and "failure to pay" as credit events, whereas CDS contracts usually also include certain forms of restructuring. For the latter credit event type, the cheapest-to-deliver option for a buyer of protection via a CDS can potentially be quite valuable, as was the case for the Conseco debt restructuring in 2000. ${ }^{13}$ For an extensive dataset of CDS quotes between 2000 and 2005, Berndt, Jarrow, and Kang (2007) report a 2.35\% median contribution of the modified-restructuring premium to total 5-year CDS spreads.

Second, index arbitrage traders are the medium by which the level of a CDS index is kept in line with so-called intrinsics, which is the fair index level implied by individual CDS spreads. Whenever intrinsics differ from the current index level, traders can profitably buy protection via the index and sell protection via the underlying CDS

\footnotetext{
${ }^{13}$ SEC filing available at www.secinfo.com/dRx61.5Yk.1.htm
} 
contracts, or vice versa. Although there are currently plans underway to automate and centralize CDS trading, the data used in this analysis covers a time period during which trading a large portfolio credit default swaps was a labor-intensive process, normally taking several hours to complete. An index arbitrage trader therefore faces significant execution risk, especially during periods of time when the index-CDS basis is volatile. Because of this risk and the cost of paying the bid-offer spread when trading individual credit default swaps, index arbitrage traders only become active when the index level differs by at least a couple of basis points from the intrinsics. As a consequence, the index-CDS basis and index-tranche basis are generally non-zero and time-varying.

To account for these effects, we relate the fixed legs $V_{i}^{\text {cds,Fixed }}$ of the underlying CDS contracts to the fixed leg $V^{\text {idx,Fixed }}$ of a credit index at time $t$ via

$$
\sum_{i=1}^{m} V_{i}^{\text {cds,Fixed }}(t)=V^{\text {idx,Fixed }}(t)\left(1+b^{\text {cds,idx }}(t)\right),
$$

where $b^{\text {cds,idx }}(t)$ is the multiplicative index-CDS basis. Similarly, we relate the tranche fixed legs to the index fixed leg via

$$
\sum_{j=1}^{J} V_{j}^{\mathrm{tr}, \text { Fixed }}(t)=V^{\mathrm{idx}, \text { Fixed }}(t)\left(1+b^{\mathrm{tr}, \mathrm{idx}}(t)\right)
$$

where $b^{\text {tr,idx }}(t)$ is the multiplicative index-tranche basis. ${ }^{14}$ From (26) and (27) it follows that the index-CDS and index-tranche basis, when measured in basis points, are given by $c_{t, M}^{\mathrm{idx}} b^{\mathrm{cds}, \mathrm{idx}}$ and $c_{t, M}^{\mathrm{idx}} b^{\mathrm{cds}, \mathrm{idx}}$, respectively, where $c_{t}^{\mathrm{idx}}$ is the index coupon at time $t$.

\subsection{Model Calibration}

This section links model-implied and mid-market CDS, credit index, and credit tranche spreads. To this end, let $c_{j, t, M}^{\mathrm{tr}}(S)$ for $S \in\{\mathrm{MI}, \mathrm{MK}\}$ denote the spread at time $t$ of the $j$ th tranche with maturity $M$ (usually 5,7 or 10 years) as implied by the model (MI), or as reported by Markit $(\mathrm{MK})$. Similarly, let $c_{i, t, M}^{\mathrm{cds}}(\mathrm{MK})$ denote the spread at time $t$ of the $i$ th CDS with maturity $M$, as reported by Markit. Finally, let $c_{t, M}^{\mathrm{idx}}(\mathrm{C})$ be the $M$-year index spread at time $t$ as reported by Citi.

We use a fitting criterion of the form ${ }^{15}$

$$
\begin{aligned}
C(\Theta)= & \omega_{\text {tr }} \operatorname{RMSE}^{\operatorname{tr}}(\Theta, \mathcal{M})^{2}+\omega_{\text {cds }} \operatorname{RMSE}^{\mathrm{cds}}(\Theta, \mathcal{M})^{2}+ \\
& \omega_{\mathrm{idx}} \operatorname{RMSE}^{\mathrm{idx}}(\Theta, \mathcal{M})^{2},
\end{aligned}
$$

\footnotetext{
${ }^{14}$ The summation on the right-hand side of (27) includes the super senior (30-100\%) tranche.

${ }^{15}$ Such a fitting criterion arises naturally in a likelihood framework with noisy observations. See Eckner (2007) for details.
} 
with weights $\omega_{\text {tr }}, \omega_{\text {cds }}$ and $\omega_{\text {idx }}$. Here, $\mathrm{RMSE}_{t}^{\mathrm{tr}}$ is the relative root mean square tranche pricing error at time $t$, defined by

$$
\operatorname{RMSE}_{t}^{\mathrm{tr}}(\Theta, \mathcal{M})=\sqrt{\frac{1}{J} \frac{1}{|\mathcal{M}|} \sum_{j=1}^{J} \sum_{M \in \mathcal{M}}\left(\frac{c_{j, t, M}^{\mathrm{tr}}(\mathrm{MI})-c_{j, t, M}^{\mathrm{tr}}(\mathrm{MK})}{c_{j, t, M}^{\mathrm{tr}}(\mathrm{MK})}\right)^{2}},
$$

$\mathrm{RMSE}_{t}^{\mathrm{cds}}$ is the relative root mean square CDS pricing error at time $t$,

$$
\operatorname{RMSE}_{t}^{\mathrm{cds}}(\Theta, \mathcal{M})=\sqrt{\frac{1}{m} \frac{1}{|\mathcal{M}|} \sum_{i=1}^{m} \sum_{M \in \mathcal{M}}\left(\frac{c_{i, t, M}^{\mathrm{cds}}(\mathrm{MI})-c_{i, t, M}^{\mathrm{cds}}(\mathrm{MK})}{c_{i, t, M}^{\mathrm{cds}}(\mathrm{MK})}\right)^{2}}
$$

and $\mathrm{RMSE}_{t}^{\mathrm{idx}}$ is the relative root mean square credit index pricing error at time $t$,

$$
\operatorname{RMSE}_{t}^{\mathrm{idx}}(\Theta, \mathcal{M})=\sqrt{\frac{1}{|\mathcal{M}|} \sum_{M \in \mathcal{M}}\left(\frac{c_{t, M}^{\mathrm{idx}}(\mathrm{MI})-c_{t, M}^{\mathrm{idx}}(\mathrm{C})}{c_{t, M}^{\mathrm{idx}}(\mathrm{C})}\right)^{2}} .
$$

The following algorithm was used for minimizing (28), that is, for fitting the basic AJD model of Section 2.1 to market-observed CDS, credit index, and credit tranche spreads:

\section{Algorithm 1}

1. For fixed parameter vector $\Theta$ and initial systematic intensity $Y_{0}$, individual $C D S$ spreads are calibrated by varying the initial intensities $\lambda_{i 0}$ for $1 \leq i \leq m$ subject to the constraint $\lambda_{i 0} \geq a_{i} Y_{0}$, and minimizing the fitting criterion (30).

2. Set $b^{c d s, i d x}=b^{t r, i d x}=0$ and determine the model implied $C D S$, index and tranche spreads. Solve for the index-CDS basis $b^{\text {cds,idx }}$ in (26), and for the index-tranche

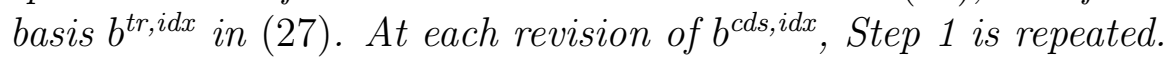

3. Vary the parameter vector $\Theta$ and the initial systematic intensity $Y_{0}$ to minimize the criterion function (28). At each revision of $\Theta$ or $Y_{0}$, Step 1 and 2 are repeated.

Step 3 was implemented by fitting each parameter separately and iterating over the set of parameters. Convergence typically occurred after 20 to 30 iterations.

\section{Results}

This section examines the fit of the model to tranche spreads of the CDX.NA.IG index at a fixed point in time, namely December 5, 2005, to facilitate comparison with Mortensen (2006). Since the emphasis of this paper are computational techniques, we focus only on a single point in time. See Eckner (2007) for a time series analysis of tranche spreads and the evolution of calibrated parameters and fitting errors over time. 


\subsection{5 year Maturity}

We start by examining the simultaneous model fit to 5-year CDS, credit index, and credit tranche spreads, using

$$
C(\Theta)=\operatorname{RMSE}_{t}^{\mathrm{tr}}(\Theta,\{5\})+5 \times \operatorname{RMSE}_{t}^{\mathrm{cds}}(\Theta,\{5\})+5 \times \operatorname{RMSE}_{t}^{\mathrm{idx}}(\Theta,\{5\})
$$

as the fitting criterion. ${ }^{16}$ Table 3 shows the model fit of Mortensen (2006) and of the model from Section 2 with deterministic recovery rates (see Example 1). For brevity we denote this model as $b A J D$. Models denoted by $b A J D_{+}$and $b A J D_{++}$additionally incorporate stochastic recovery rates (see Example 2), and stochastic and serially correlated recovery rates (see Appendix $\mathrm{C}$ ), respectively.

\begin{tabular}{l|rr|rrrrrr}
\hline \hline Tranche & Bloomberg & Mort & Markit & Citi & MS & bAJD & bAJD + & bAJD $_{++}$ \\
\hline $0 \%-3 \%$ & $41.1 \%$ & $43.2 \%$ & $40.7 \%$ & $40.8 \%$ & $41.1 \%$ & $40.2 \%$ & $39.8 \%$ & $40.2 \%$ \\
$3 \%-7 \%$ & 117.5 & 125.9 & 111.9 & 112.5 & 113.5 & 121.7 & 128.6 & 122.1 \\
$7 \%-10 \%$ & 32.9 & 30.6 & 31.3 & 31.3 & 31.0 & 28.5 & 28.0 & 30.0 \\
$10 \%-15 \%$ & 15.8 & 21.3 & 13.5 & 13.5 & 14.5 & 14.6 & 14.8 & 14.4 \\
$15 \%-30 \%$ & 7.0 & 8.8 & 7.4 & 7.5 & 7.3 & 7.2 & 7.2 & 7.2 \\
\hline $\operatorname{RMSE}^{\text {tr }}(\Theta, 5)$ & - & 0.200 & - & - & - & 0.069 & 0.093 & 0.062 \\
$\operatorname{RMSE}^{\mathrm{cds}}(\Theta, 5)$ & - & - & - & - & - & 0.000 & 0.000 & 0.000 \\
$\operatorname{RMSE}^{\text {idx }}(\Theta, 5)$ & - & - & - & - & - & 0.000 & 0.000 & 0.000 \\
\hline \hline
\end{tabular}

Table 3: Comparison of the fit to tranche spreads on December 5, 2005, by different models. Here, Mort is the model by Mortensen (2006), which was fitted to prices from the Bloomberg system, while $b A J D$ is the model from Section 2.1 with deterministic recovery rates and was fitted to data provided by Markit. Models $b A J D_{+}$and $b A J D_{++}$in addition incorporate stochastic recovery rates, and stochastic and serially correlated recovery rates, respectively.

Table 3 shows considerable dispersion in tranche mid-market prices across the data providers Bloomberg, Markit, Citi and Morgan Stanley, probably reflecting the typical difficulty of price discovery in an over-the-counter market. Regarding the model fit, in general all models seem to give a reasonable fit to market-observed tranche spreads on December 5, 2005, except for the junior mezzanine (3-7\%) tranche. This pattern is not unique to stochastic intensity models, but also tends to show up with other pricing models in use. Conversations with practitioners suggest that this effect might be due to supply-demand imbalances. At that time, investment banks bought a lot of bespoke mezzanine protection from clients, and hedged the resulting short position using standardized credit tranches, which led to downward pressure on the spread of this tranche.

\footnotetext{
${ }^{16}$ Since the super senior (30-100\%) tranche was not actively traded in the period of investigation, it is excluded from our analysis.
} 
The bottom three rows in Table 3 shows the root mean square tranche pricing errors (29)-(31). We see that the more flexible correlation structure of the model from Section 2 makes it possible to improve the fit of Mortensen (2006) by a factor of around three in terms of RMSE. We also see that for this particular date, the model with deterministic recovery rates $(b A J D)$ is able to more closely fit market-observed tranche spreads than the model with stochastic recovery rates $\left(b A J D_{+}\right)$, even though the latter model better matches the historical behavior of recovery rates and was therefore a priori expected to give a better fit. Stochastic recovery rates introduce additional tail risk into the model, which causes the model-implied spread of the 3-7\% tranche to increase since its lower attachment point already lies above the expected portfolio loss amount over a five-year time horizon. This effect reduces the ability of model with stochastic recovery rates to fit the relatively low spread of the junior mezzanine tranche, and therefore increases the root mean square pricing error.

Finally, even though the model with stochastic and serially correlated recovery rates $\left(b A J D_{++}\right)$best fits tranche spreads in terms of the root mean square tranche pricing error (29), the fitted recovery rate dynamics are not realistic. The transition probabilities $\rho_{1}, \ldots, \rho_{m}$ in $(50)$ were allowed to vary freely in $[0,1]$, and ended up taking on the values 0 and 1 most of the time. The purpose of this model is merely to illustrate the size of the potential fitting improvement, by switching from deterministic recovery rates to a more flexible class of recovery rate scenarios.

\subsection{All Maturities}

We next examine the model, when simultaneously fit to 5, 7 and 10-year CDS, credit index, and tranche spreads, when using the basic AJD model with deterministic recovery rates, and using

$$
\begin{aligned}
C(\Theta)= & \operatorname{RMSE}_{t}^{\mathrm{tr}}(\Theta,\{5,7,10\})+5 \times \operatorname{RMSE}_{t}^{\mathrm{cds}}(\Theta,\{5,7,10\}) \\
& +5 \times \operatorname{RMSE}_{t}^{\operatorname{idx}}(\Theta,\{5,7,10\})
\end{aligned}
$$

as the fitting criterion. Table 4 shows that this model captures the term-structure of 5 , 7 and 10-year tranche spreads rather well, again except for the junior mezzanine (3-7\%) tranche. The tranche-RMSE is 0.083 , and senior tranche spreads are fit almost perfectly for all available maturities. With a RMSE of 0.046, the term-structure of CDS spreads is fit well. The same holds true for the fit to credit index spreads, with an RMSE of 0.003 .

\subsection{Parameter Estimates}

Table 5 reports the fitted parameters for the basic AJD model with deterministic, stochastic, and stochastic and serially correlated recovery rates. The first three rows 


\begin{tabular}{l|rr|rr|rr}
\hline \hline Tranche & Mkt 5yr & Model 5yr & Mkt 7yr & Model 7yr & Mkt 10yr & Model 10yr \\
\hline $0 \%-3 \%$ & $40.7 \%$ & $40.0 \%$ & $54.8 \%$ & $53.4 \%$ & $61 \%$ & $58.8 \%$ \\
$3 \%-7 \%$ & 111.9 & 123.2 & 270.5 & 312.3 & 647 & 666 \\
$7 \%-10 \%$ & 31.3 & 27.6 & 53.5 & 55.6 & 129 & 138 \\
$10 \%-15 \%$ & 13.5 & 15.6 & 29.8 & 29.6 & 65 & 54 \\
$15 \%-30 \%$ & 7.4 & 7.4 & 11.6 & 11.3 & 23 & 24 \\
\hline Index & 49 & 48.9 & 58 & 58.3 & 71 & 70.8 \\
\hline RMSE $^{\text {tr }}(\Theta, 5,7,10)$ & \multicolumn{7}{|c}{} & & & 0.083 \\
$\operatorname{RMSE}^{\operatorname{cds}}(\Theta, 5,7,10)$ & \multicolumn{7}{|c}{} & & & 0.046 \\
$\operatorname{RMSE}^{\text {idx }}(\Theta, 5,7,10)$ & \multicolumn{7}{|c|}{} & & & & 0.003 \\
\hline \hline
\end{tabular}

Table 4: Model fit of the basic AJD model with deterministic recovery rates to 5,7 and 10-year tranche spreads on December 5, 2005.

show the fitted parameters when using only 5-year tranche spreads. As expected, the estimates are somewhat unstable and depend on the assumed recovery rate dynamics. For example, the effect of an increase in the diffusive drift $\kappa_{Y} \theta_{Y}$ of the systematic factor can be approximately offset by a decrease in the "jump drift" $l_{Y} \mu_{Y}$, so that the parameters cannot be accurately identified with 5-year data alone.

\begin{tabular}{l|rrrrrrrrrr}
\hline \hline & $k$ & $k \tilde{\theta}_{\text {Avg }} \times 10^{4}$ & $\sigma$ & $\widetilde{l}$ & $\widetilde{l} \mu \times 10^{4}$ & $\omega_{1}$ & $\omega_{2}$ & $Y_{0} \times 10^{4}$ & $b^{\text {cds,idx }}$ & $b^{\text {tr,idx }}$ \\
\hline bAJD $(5)_{\text {bAJD }_{+}(5)}$ & 0.045 & 0.22 & 0.103 & 0.010 & 30.3 & 0.22 & 0.05 & 12.7 & 0.031 & 0.137 \\
bAJD $_{++}(5)$ & 0.058 & 0.29 & 0.104 & 0.010 & 30.2 & 0.23 & 0.05 & 12.0 & 0.031 & 0.135 \\
\hline bAJD $(5,7,10)$ & -0.087 & 0.24 & 0.105 & 0.010 & 29.8 & 0.24 & 0.05 & 12.6 & 0.031 & 0.139 \\
bAJD $_{+}(5,7,10)$ & -0.214 & 1.07 & 0.068 & 0.007 & 21.7 & 0.36 & 0.28 & 5.2 & 0.024 & 0.083 \\
bAJD $_{++}(5,7,10)$ & -0.214 & 1.07 & 0.068 & 0.007 & 21.7 & 0.36 & 0.28 & 4.9 & 0.024 & 0.076 \\
\hline \hline
\end{tabular}

Table 5: Fitted model parameters for December 5, 2005. $b A J D$ is the model from Section 2.1 with deterministic recovery rates, while models $b A J D_{+}$and $b A J D_{++}$in addition incorporate stochastic recovery rates, and stochastic and serially correlated recovery rates, respectively. The numbers in parenthesis following the model name indicate the tranche maturities to which the model was fitted.

In contrast, when fitting the whole term-structure of tranche spreads, risk-neutral parameter estimates are stable, as can be seen from the last three rows of Table 5 . We therefore concentrate on interpreting these model parameters. First of all, we see that default intensities are explosive, i.e. have negative risk-neutral mean-reversion rates. The parameter $\sigma=0.068$ implies a proportional default intensity volatility of $96 \%$ per year for a firm with common factor loading equal to one and initial default intensity of 50 basis points. With $\widetilde{l}=0.007$, jumps in default intensities are relatively rare, with an average of slightly less than one jump per year in a portfolio of 125 companies. The risk-neutral expected jump size is large, at around 3000 basis points. In view of 
the accounting scandals of 2001-2002, and the 2007-2008 credit crisis, these risk-neutral parameters do not seem to be excessive. With $\omega_{1}=0.36$, about a third of the jumps are economy-wide events, while two thirds of the jumps are events that affect only a single company. With

$$
\omega_{2}=\frac{\theta_{Y}}{\widetilde{\theta}_{\mathrm{Avg}}}=0.28>\frac{Y_{0}}{\operatorname{Avg}\left(\lambda_{i 0}\right)}=0.14,
$$

investors take a more pessimistic view about the future evolution of the systematic factor than about the idiosyncratic factors. In other words, the ratio of $Y$ to individual default intensities exhibits an upward-sloping term-structure. A value of $b^{\text {cds,idx }}=0.024$ implies an index-CDS basis of less than two basis points for all maturities, while $b^{\text {tr,idx }}=0.083$ implies an index-tranche basis of 4-6 basis points for all maturities.

\subsection{Hedging}

This section examines the calculation of various hedging ratios, which is important for the usefulness of a model in practice. For example, a financial institution who enters a credit derivative contract with a client, usually wants to limit its exposure to various types risks.

\subsubsection{Traditional Deltas}

We start by examining the problem of using a credit index contract to hedge a credit tranche against market-wide changes in credit conditions. To this end, we fix a point in time $t$ and credit index maturity $M$ for the remainder of this section. To shorten notation, let $\operatorname{Idx}(\Theta)=c_{t, M}^{\mathrm{idx}}(\Theta)$ denote the model-implied credit index spread, $\operatorname{Tr}_{j}(\Theta)=$ $c_{j, t, M}^{\mathrm{tr}}(\Theta)$ the model-implied spread of the $j$ th tranche, and $\operatorname{CDS}_{i}(\Theta)=c_{i, t, M}^{\mathrm{cds}}(\Theta)$ the model-implied CDS spread of the $i$ th issuer in the CDX portfolio.

A priori, it is not clear how to define hedging ratios in the basic AJD framework of Section 2.1, because there are multiple sources of risk in the model. For estimating the effect of a uniform, market-wide increase in credit spreads, we consider a family of models parameterized by a fixed common scaling of all risk-neutral intensity processes by the same positive constant $\varepsilon$. We define the index delta $\Delta_{j}^{\mathrm{idx}}(t)$, which measures the price sensitivity of the $j$ th tranche with respect to the credit index for market-wide changes in credit conditions, as

$$
\Delta_{j}^{\mathrm{idx}}(t)=\left.\frac{\partial \operatorname{Tr}_{j}(\Theta)}{\partial \varepsilon}\right|_{\varepsilon=1} /\left.\frac{\partial \operatorname{Idx}(\Theta)}{\partial \varepsilon}\right|_{\varepsilon=1} .
$$

The derivatives can be calculated numerically. 
Similarly, consider a family of models parameterized, for a given firm $i$, by a positive scaling $\varepsilon_{i}$ of the $i$ th firm's risk-neutral intensity process $\lambda_{i}$. We define the single-name delta $\Delta_{j}^{(i)}(t)$, measuring the price sensitivity of the $j$ th tranche with respect to changes in the credit quality of the $i$ th name, as

$$
\Delta_{j}^{(i)}(t)=\left.\frac{\partial \operatorname{Tr}_{j}(t, T, \Theta)}{\partial \varepsilon_{i}}\right|_{\varepsilon_{i}=1} /\left.\frac{\partial \operatorname{CDS}_{i}(t, T, \Theta)}{\partial \varepsilon_{i}}\right|_{\varepsilon_{i}=1}
$$

For December 5, 2005, Table 6 provides 5-year tranche deltas $\Delta_{j}^{\text {idx }}(t)$ with respect to the index for the fitted basic AJD models from Section 5.1. The table also provides, for comparison, deltas implied by the Gaussian copula model, which are obtained by "bumping" CDS spreads. The deltas of the models are quite similar, so that the $\Delta$ based hedging performances of these models will be comparable. ${ }^{17}$

\begin{tabular}{l|rrrrr}
\hline \hline & $0 \%-3 \%$ & $3 \%-7 \%$ & $7 \%-10 \%$ & $10 \%-15 \%$ & $15 \%-30 \%$ \\
\hline$\Delta_{j, \text { copula }}$ & 18.5 & 5.5 & 1.5 & 0.8 & 0.4 \\
$\Delta_{j, \mathrm{bx}}^{\text {idx }}$ & 22.9 & 6.9 & 1.1 & 0.4 & 0.2 \\
$\Delta_{j, \mathrm{~b}}^{\mathrm{idx}}$ & 23.0 & 6.8 & 1.0 & 0.4 & 0.3 \\
$\Delta_{j, \mathrm{bx}}^{\text {idx }}$ & 22.9 & 6.9 & 1.0 & 0.4 & 0.3 \\
\hline \hline
\end{tabular}

Table 6: Five-year tranche deltas with respect to the underlying credit index as implied by (i) the copula model and (ii) the fitted basic AJD models $b A J D(5,7,10), b A J D_{+}(5,7,10)$ and $b A J D_{++}(5,7,10)$ from Section 5.1. Data are for the 5-year CDX.NA.IG index on December 5, 2005.

Table 7 reports the single-name deltas (33) for the first couple of firms in the CDX.NA.IG portfolio on December 5, 2005. Comparing the deltas across companies, we see that credit spread changes of the most risky names in the portfolio have a relatively larger impact on the equity tranche, whereas credit spread changes of low-risk names have a relatively larger impact on the senior tranches. To understand this effect, it is helpful to consider the likely ordering of defaults in the presence of a systematic risk factor; the default of an already risky credit will most likely be an idiosyncratic event and therefore mostly affect the equity tranche, while the default of a currently high-quality name will more likely be a systematic event - in which many companies default over a short period of time - and therefore more likely affect the senior tranches too.

\footnotetext{
${ }^{17}$ Repeating the calculation of deltas for the first day of each month between September 2004 and November 2006 gave similar hedging ratios.
} 


\begin{tabular}{l|r|rrrrr}
\hline \hline & & \multicolumn{5}{|c}{ Single-name Tranche Delta $\times 125$} \\
Company & 5yr CDS & $0 \%-3 \%$ & $3 \%-7 \%$ & $7 \%-10 \%$ & $10 \%-15 \%$ & $15 \%-30 \%$ \\
\hline Alcoa Inc. & 28 & 20.8 & 6.3 & 1.1 & 0.4 & 0.4 \\
Albertsons Inc. & 306 & 24.3 & 5.3 & 0.5 & 0.1 & 0.0 \\
ACE Ltd & 28 & 20.8 & 6.3 & 1.1 & 0.4 & 0.4 \\
Amern Elec Pwr Co Inc & 40 & 21.8 & 6.4 & 1.1 & 0.4 & 0.4 \\
Aetna Inc. & 23 & 20.3 & 6.2 & 1.1 & 0.5 & 0.4 \\
Amern Intl Gp Inc & 19 & 19.9 & 6.1 & 1.1 & 0.5 & 0.4 \\
\hline \hline
\end{tabular}

Table 7: Five-year tranche deltas with respect to individual credits in the underlying portfolio as implied by the fitted bAJD $(5,7,10)$ model from Section 5.1. Data are for the first six companies of the 5-year CDX.NA.IG index on December 5, 2005.

\subsubsection{Higher-Order Risks}

"Higher-order" risks in the credit markets include, for example, changes in correlations, volatilities, or idiosyncratic default risk. ${ }^{18}$ For the basic AJD model, deltas with respect to these risks can be computed in a manner analogous to (32). Such effects cannot be considered in the inherently static copula model. As an example, Table 8 shows the tranche deltas

$$
\Delta_{j}^{X_{i}}(t)=\frac{\partial \operatorname{Tr}_{j}(\Theta)}{\partial X_{i 0}} / \frac{\partial \operatorname{Idx}(\Theta)}{\partial X_{i 0}}
$$

with respect to changes in the initial value of the idiosyncratic default risk factor $X_{i}$ of firm $i$. This factor captures, for example, the risk of a leveraged buy-out, the outcome of a clinical trial, or the unexpected resignation of the firm's CEO, all of which are unlikely to affect other companies in a material way. Table 8 shows that, as expected, idiosyncratic shocks to a firm's credit quality affect mainly the equity tranche and have hardly any effect on senior tranche spreads. Moreover, the delta of a firm with a high credit spread tends to be smaller than the delta of a firm with low credit spread; if a company is likely to default during the next couple of years, an idiosyncratic shock to its default intensity has a relatively smaller impact, since the firm cannot default more than once.

\section{Conclusion}

We presented computational techniques that enhance the applicability of a multivariate intensity-based model of corporate defaults, along the lines of Duffie and Gârleanu (2001) and Mortensen (2006), for pricing of structured credit derivatives. We showed

\footnotetext{
${ }^{18}$ Due to the doubly stochastic assumption, certain risk sources, such as contagion, cannot be captured in this framework, even though it would be desirable in light of the 2007-2008 financial crisis.
} 


\begin{tabular}{l|r|rrrrr}
\hline \hline & & \multicolumn{5}{|c}{ Idiosyncratic Tranche Delta $\times 125$} \\
Company & 5yr CDS & $0 \%-3 \%$ & $3 \%-7 \%$ & $7 \%-10 \%$ & $10 \%-15 \%$ & $15 \%-30 \%$ \\
\hline Alcoa Inc. & 28 & 26.6 & 6.2 & 0.3 & 0.1 & 0.0 \\
Albertsons Inc. & 306 & 25.8 & 4.7 & 0.2 & 0.0 & 0.0 \\
ACE Ltd & 28 & 26.6 & 6.2 & 0.3 & 0.1 & 0.0 \\
Amern Elec Pwr Co Inc & 40 & 26.6 & 6.1 & 0.3 & 0.1 & 0.0 \\
Aetna Inc. & 23 & 26.5 & 6.2 & 0.3 & 0.1 & 0.0 \\
Amern Intl Gp Inc & 19 & 26.5 & 6.2 & 0.3 & 0.1 & 0.0 \\
\hline \hline
\end{tabular}

Table 8: Five-year tranche deltas with respect to the initial values $X_{i 0}$ of the idiosyncratic risk factors $X_{i}$ as implied by the fitted $b A J D(5,7,10)$ model from Section 5.1. Data are for the first six companies of the 5-year CDX.NA.IG index on December 5, 2005.

that the computational tractability of such a model is similar to that of the Gaussian copula model, since both model implementations have the recursive calculation of the conditional portfolio loss distribution via the Andersen-Sidenius-Basu algorithm as the computational bottleneck.

We improved upon the model fit in Mortensen (2006) by a factor of around three, by allowing for a more flexible correlation structure, and by accounting for market frictions due to bid-offer spreads. We showed that a rich class of recovery rate scenarios can be incorporated into the model in a computationally tractable manner. The resulting model can be used to hedge a wide range of risks in the credit market, such as the risk of changes in correlations, volatilities, or idiosyncratic default risk - not all of which can be considered in the inherently static copula model.

We hope that our work spurs additional research in the area of bottom-up models for portfolio credit risk, which are required for consistently pricing credit derivatives that either fully or partially share each other's underlying portfolio, such as bespoke CDOs. For example, more than one common factor could be incorporate into the basic AJD model to capture the correlation structure at the sectoral level. In this case, survival probabilities would still be known in closed-form, but calculating the portfolio loss distribution via (18) would require multi-dimensional numerical integration which is computationally more burdensome. For securities with long time horizons, the assumption of constant volatility is often fairly innocuous due to the central limit theorem. However, for the pricing of short-dated securities, like options on credit indices and tranches, having a model that incorporates stochastic volatility and jumps in volatility would be desirable. Since evaluating the characteristic function (4) of an integrated AJD accounts for less than $8 \%$ of the total computing time in our implementation, more elaborate affine processes (supporting stochastic volatility, time-varying jump intensities, multiple factors) could be used as the factors driving default intensities, even if such an extension requires the ODEs in (36) to be solved numerically. Although we have examined stochastic and serially correlated recovery rates, it would be desirable to also incorporate countercyclical 
recovery rates, which are empirically well-documented and potentially quite important for pricing of credit derivatives that are heavily exposed to tail risk. 


\section{Appendices}

\section{A Basic Affine Jump Diffusions}

This section lists a couple of useful properties about basic Affine Jump Diffusions. It builds on Duffie, Pan, and Singleton (2000), Duffie and Gârleanu (2001), and Appendix A of Duffie and Singleton (2003). For a more general treatment of affine processes see Duffie, Filipović, and Schachermayer (2003).

A stochastic process $X$ on a filtered probability space $\left(\Omega, \mathcal{F},\left(\mathcal{F}_{t}\right), \mathbb{P}\right)$ is called a basic AJD, or short-hand $X=\operatorname{bAJD}\left(x_{0}, \kappa, \theta, \sigma, l, \mu\right)$, if its dynamics are of the form

$$
d X_{t}=\kappa\left(\theta-X_{t}\right) d t+\sigma \sqrt{X_{t}} d B_{t}+d J_{t}, \quad X_{0}=x_{0},
$$

where $B$ is a standard Brownian motion, and $J$ is an independent compound Poisson process with jump intensity $l$ and exponentially distributed jumps with mean $\mu$. The moment generating function of the jump size distribution $\nu$ is

$$
\psi(c)=\int_{\mathbb{R}} e^{c z} d \nu(z)=\frac{1}{1-c \mu},
$$

for $c \in \mathbb{C}$ and $\operatorname{Re}(c)<1 / \mu$.

\section{A.1 Moment Generating Function}

Let $X$ be a basic AJD with dynamics given by (34). From Proposition 1 in Duffie, Pan, and Singleton (2000) it follows that for $t>0$ and $q \in \mathbb{R}$

$$
E\left(e^{q \int_{0}^{t} X_{s} d s}\right)=e^{\alpha(t)+\beta(t) X_{0}},
$$

where $\alpha(\cdot)$ and $\beta(\cdot)$ solve the pair of Riccati ordinary differential equations

$$
\begin{aligned}
& \alpha^{\prime}(t)=-\kappa \theta \beta(t)-l(\psi(\beta(t))-1) \\
& \beta^{\prime}(t)=\kappa \beta(t)-\frac{1}{2} \sigma^{2} \beta(t)^{2}-q,
\end{aligned}
$$

with boundary conditions $\alpha(0)=\beta(0)=0$.

Duffie and Gârleanu (2001) give an explicit solution for $\alpha$ and $\beta$ for a slightly more general transform than (35). In our case, their formula simplifies to

$$
\begin{aligned}
\alpha(t)= & -\frac{2 \kappa \theta}{\sigma^{2}} \log \left(\frac{c_{1}+d_{1} e^{-\gamma t}}{c_{1}+d_{1}}\right)+\frac{\kappa \theta}{c_{1}} t+ \\
& +l\left(\frac{d_{1} / c_{1}-d_{2} / c_{2}}{-\gamma d_{2}}\right) \log \left(\frac{c_{2}+d_{2} e^{-\gamma t}}{c_{2}+d_{2}}\right)+l \frac{1-c_{2}}{c_{2}} t \\
\beta(t)= & \frac{1-e^{-\gamma t}}{c_{1}+d_{1} e^{-\gamma t}},
\end{aligned}
$$


where

$$
\begin{aligned}
& \gamma=\sqrt{\kappa^{2}-2 \sigma^{2} q} \\
& c_{1}=(\kappa+\gamma) /(2 q) \\
& c_{2}=1-\mu / c_{1} \\
& d_{1}=(-\kappa+\gamma) /(2 q) \\
& d_{2}=\left(d_{1}+\mu\right) / c_{1}
\end{aligned}
$$

Hence, the moment generating function of an integrated basic AJD,

$$
\widetilde{X}_{t} \equiv \int_{0}^{t} X_{s} d s,
$$

is known in closed-form.

\section{A.2 Characteristic Function}

Again let $X$ be a basic AJD with dynamics given by (34). Setting $q=i u$ for $u \in \mathbb{R}$ in (35) gives an explicit formula for the characteristic function of the integrated AJD (39). Although Duffie and Gârleanu (2001) originally solved (36) and arrived at (37) only for real-valued $q$, the derivation can be repeated for the complex-valued version of (36). In this case, we interpret $\gamma$ in (38) as

$$
\gamma=\left|\gamma^{2}\right|^{1 / 2} \exp \left(i \arg \left(\gamma^{2}\right) / 2\right)
$$

where for any $z \in \mathbb{C}, \arg (z)$ is defined such that $z=|z| \exp (i \arg (z))$ with $-\pi<$ $\arg (z) \leq \pi$. Moreover, we take $\log (z)=\log (|z|)+i \arg (z)$, although any other branch of the complex logarithm would work as well, since the logarithm of $\gamma$ shows up only in the exponent of (35). See Lord and Kahl (2006) for a discussion on evaluating transforms of the form (35) with complex-valued exponent.

\section{B Pricing}

This section gives a detailed description of the CDS, credit index and tranche pricing mechanics, not just for the basic AJD framework of Section 2, but for any model of portfolio credit risk for which Assumption 1 holds. The pricing formulas are similar to the ones in Berndt, Douglas, Duffie, Ferguson, and Schranz (2005), Mortensen (2006), and Errais, Giesecke, and Goldberg (2006), but include a more detailed treatment of day counting conventions and accrual payments. 


\section{B.1 CDS Pricing}

For a fixed point in time $t$, consider a credit default swap written on a reference entity $i$, with maturity $M$, and notional amount $N_{i}$. Let $c_{i, t, M}^{\text {cds }}$ denote the CDS spread at time $t$, and $\left\{t_{l}: 1 \leq l \leq n\right\}$ the set of coupon payment dates. The market value of the payments made by the buyer of protection (commonly called the fixed leg) equals

$$
\begin{aligned}
V_{i}^{\text {cds,Fixed }}(t)= & E_{t}^{\mathbb{Q}}\left(\sum_{\left\{l: t_{l}>t\right\}} e^{-\int_{t}^{t_{l}} r_{s} d s} 1_{\left\{\tau_{i}>t_{l}\right\}} N_{i} c_{i, t, M}^{\mathrm{cds}} \frac{\left(t_{l}-t_{l-1}\right)}{360}\right) \\
& -N_{i} c_{i, t, M}^{\mathrm{cds}} \frac{t-\max \left(t_{l}: t_{l} \leq t\right)}{360}
\end{aligned}
$$

using an Actual/360 day-count convention, where $\tau_{i}$ is the default time of company $i$ and where the risk-neutral expectation is taken conditional on all available information up to time $t$. The second term in (40) reflects the accrued premium between the most recent coupon payment date and the time of entering the CDS contract.

The market value of the payments made by the seller of protection (commonly called the protection leg) equals

$$
V_{i}^{\mathrm{cds}, \operatorname{Prot}}(t)=E_{t}^{\mathbb{Q}}\left(e^{-\int_{t}^{\tau_{i}} r_{s} d s} 1_{\left\{\tau_{i} \leq t_{n}\right\}} W_{\tau_{i}}\right)
$$

where

$$
W_{\tau_{i}}=N_{i}\left(1-R_{i}\right)-N_{i} c_{i, t, M}^{\mathrm{cds}}\left(\tau_{i}-\max \left(t_{l}: t_{l} \leq \tau_{i}\right)\right)
$$

is the payment made in case of default at time $\tau_{i}$, and $R_{i}$ denotes the possibly random recovery rate. The second term in (42) reflects the accrued premium at the time of default.

Under Assumption 1, the market value of the fixed leg (40) can be approximated as

$$
\begin{aligned}
V_{i}^{\text {cds, Fixed }}(t) \approx & N_{i} c_{i, t, M}^{\text {cds }} \sum_{\left\{l: t_{l}>t\right\}} B_{t}\left(t_{l}\right) \frac{\left(t_{l}-t_{l-1}\right)}{360} \mathbb{Q}_{t}\left(\tau_{i}>t_{l}\right) \\
& -N_{i} c_{i, t, M}^{\text {cds }} \frac{t-\max \left(t_{l}: t_{l} \leq t\right)}{360}
\end{aligned}
$$

where $B_{t}(T)$ is the discount function at time $t$ for a unit payoff at maturity $T \geq t$, and where $\mathbb{Q}_{t}(A)$ denotes the risk-neutral probability of an event $A$, conditional on all available information up to time $t$.

Using the approximation that defaults occur half-way in between coupon payment dates, the market value of the protection leg (41) can be approximated as 


$$
\begin{aligned}
V_{i}^{\text {cds,Prot }}(t) \approx & N_{i} \sum_{\left\{l: t_{l}>t\right\}} B_{t}\left(\frac{\max \left(t_{l-1}, t\right)+t_{l}}{2}\right) \mathbb{Q}_{t}\left(\tau_{i} \in\left(\max \left(t_{l-1}, t\right), t_{l}\right]\right) \\
& \times\left[\left(1-E^{\mathbb{Q}}\left(R_{i}\right)\right)-\frac{c_{i, t, M}^{\mathrm{cds}}}{2} \frac{\left(t_{l}-\max \left(t_{l-1}, t\right)\right)}{360}\right],
\end{aligned}
$$

where we used that, under Assumption 1 , the recovery rate $R_{i}$ is $\mathbb{Q}$-independent of all other random variables in the model, so that due to the tower property, $R_{i}$ can without loss of generality be treated as a constant and equal to its risk-neutral expectation.

The fair CDS spread is obtained by setting (43) equal to (44) and solving for $c_{i, t, m}^{\mathrm{cds}}$. As shown in Section 3 , the quantities $\mathbb{Q}_{t}\left(\tau_{i}>t_{l}\right)$ and therefore also

$$
\mathbb{Q}_{t}\left(\tau_{i} \in\left(t_{l-1}, t_{l}\right]\right)=\mathbb{Q}_{t}\left(\tau_{i}>t_{l-1}\right)-\mathbb{Q}_{t}\left(\tau_{i}>t_{l}\right)
$$

are known in closed-form in the basic AJD framework of Section 2. Model-implied CDS spreads are therefore also known in closed-form.

\section{B.2 Index Pricing}

For a fixed point in time $t$, consider a credit index contract with maturity $M$, index spread $c_{t, M}^{\mathrm{idx}}$, and with $m$ companies in the underlying portfolio. By market convention, the remaining notional size of the index contract at a time $s \geq t$ equals

$$
N_{s}^{\mathrm{idx}}=\sum_{i=1}^{m} N_{i} \mathbf{1}_{\left\{\tau_{i}>s\right\}},
$$

where $N_{i}$ is the notional exposure of the index to company $i$ in the underlying portfolio.

Under Assumption 1, the market value of the fixed-leg can be approximated as

$$
\begin{aligned}
V^{\mathrm{idx}, \text { Fixed }}(t) \approx & c_{t, M}^{\mathrm{idx}} \sum_{\left\{l: t_{l}>t\right\}} B_{t}\left(t_{l}\right) \frac{\left(t_{l}-t_{l-1}\right)}{360} E_{t}^{\mathbb{Q}}\left(N_{t_{l}}^{\mathrm{idx}}\right) \\
& -N c_{t, M}^{\mathrm{idx}} \frac{t-\max \left(t_{l}: t_{l} \leq t\right)}{360}
\end{aligned}
$$

where the second term reflects the accrued premium between the most recent coupon payment date and the time of entering the index contract.

Using the approximation that defaults occur half-way in between coupon payment dates, the market value of the payments made by the protection leg can be approximated 
as

$$
\begin{aligned}
V^{\mathrm{idx}, \operatorname{Prot}}(t) \approx & \sum_{\left\{l: t_{l}>t\right\}} B_{t}\left(\frac{\max \left(t_{l-1}, t\right)+t_{l}}{2}\right) E_{t}^{\mathbb{Q}}\left(N_{t_{l}}^{\mathrm{idx}}-N_{\max \left(t_{l-1}, t\right)}^{\mathrm{idx}}\right) \\
& \times\left[\left(1-E^{\mathbb{Q}}\left(R_{i}\right)\right)-\frac{c_{t, M}^{\mathrm{idx}}}{2} \frac{\left(t_{l}-\max \left(t_{l-1}, t\right)\right)}{360}\right],
\end{aligned}
$$

where the second term in the brackets reflects the expected accrual payment at the time of default.

The fair index spread is obtained by setting (46) equal to (47) and solving for $c_{t, M}^{\text {idx }}$. Since the quantities $\mathbb{Q}_{t}\left(\tau_{i}>t_{l}\right)$ and therefore also $\mathbb{Q}_{t}\left(\tau_{i} \in\left(t_{l-1}, t_{l}\right]\right)$ and

$$
E_{t}^{\mathbb{Q}}\left(N_{t_{l}}^{\mathrm{idx}}\right)=\sum_{i=1}^{m} N_{i} \mathbb{Q}_{t}\left(\tau_{i}>t_{l}\right)
$$

are known in closed-form in the basic AJD framework of Section 2, model-implied index spreads are also known in closed-form.

\section{B.3 Tranche Pricing}

For a fixed point in time $t$, consider a credit tranche with maturity $M$, tranche spread $c_{j, t, M}^{\mathrm{tr}}$, and lower and upper attachment point $\underline{A}_{j}$ and $\bar{A}_{j}$, respectively. For the purpose of determining the cash flows of a tranche, the market has adopted a slightly different definition of the portfolio notional than (45) for credit indices, namely for $s \geq t$,

$$
N_{s}^{\mathrm{tr}}=\left(\sum_{i=1}^{m} N_{i}\right)-L_{s}^{\mathrm{tr}},
$$

where

$$
L_{s}^{\mathrm{tr}}=\sum_{i=1}^{m} N_{i}\left(1-R_{i}\right) \mathbf{1}_{\left\{\tau_{i} \leq s\right\}}
$$

denotes the portfolio loss amount up to time $s$. The notional size of tranche $j$ at time $s$ is defined as

$$
N_{j, s}^{\mathrm{tr}}=N\left(\bar{A}_{j}-\underline{A}_{j}\right)-\left[\max \left(L_{s}^{\mathrm{tr}}-\underline{A}_{j} N, 0\right)-\max \left(L_{s}^{\mathrm{tr}}-\bar{A}_{j} N, 0\right)\right],
$$

which is reminiscent of an option spread on the portfolio loss amount. ${ }^{19}$

\footnotetext{
${ }^{19}$ The definition of the remaining notional size for the super senior $(30 \%-100 \%)$ tranche is slightly different, namely $0.7 N-\left[\left(L_{s}^{\mathrm{tr}}-0.3 N\right)^{+}-\left(L_{s}^{\mathrm{tr}}-0.7 N\right)\right]-\left(\frac{N}{m} \sum \mathbf{1}_{\left\{\tau_{i} \leq s\right\}}-L_{s}^{\mathrm{tr}}\right)$.
} 
Under Assumption 1, the market value of the fixed-leg of the $j$ th tranche can be approximated as

$$
\begin{aligned}
V_{j}^{\mathrm{tr}, \text { Fixed }}(t) \approx & c_{j, t, M}^{\mathrm{tr}} \sum_{\left\{l: t_{l}>t\right\}} B_{t}\left(t_{l}\right) \frac{\left(t_{l}-t_{l-1}\right)}{360} E_{t}^{\mathbb{Q}}\left(N_{j, t_{l}}^{\mathrm{tr}}\right) \\
& -N_{j, t}^{\mathrm{tr}} c_{j, t, M}^{\mathrm{tr}} \frac{t-\max \left(t_{l}: t_{l} \leq t\right)}{360}
\end{aligned}
$$

where the second term reflects the accrued premium between the most recent coupon payment date and the time of entering the tranche contract.

Using the approximation that defaults occur half-way in between coupon payment dates, the market value of the protection leg can be approximated as

$$
\begin{aligned}
V_{j}^{\mathrm{tr}, \operatorname{Prot}}(t) \approx & \sum_{\left\{l: t_{l}>t\right\}} B_{t}\left(\frac{\max \left(t_{l-1}, t\right)+t_{l}}{2}\right) E_{t}^{\mathbb{Q}}\left(N_{j, t_{l}}^{\mathrm{tr}}-N_{j, \max \left(t_{l-1}, t\right)}^{\mathrm{tr}}\right) \\
& \times\left[1-\frac{c_{j, t, M}^{\mathrm{tr}}}{2} \frac{\left(t_{l}-\max \left(t_{l-1}, t\right)\right)}{360}\right],
\end{aligned}
$$

where the second term in the brackets reflects the expected accrual payment at the time of default.

The fair tranche spread is obtained by setting (48) equal to (49) and solving for $c_{j, t, M}^{\mathrm{tr}}$. Under Assumption 1, the quantities $E_{t}^{\mathbb{Q}}\left(N_{j, t_{l}}^{\mathrm{tr}}\right)$ and therefore also

$$
E_{t}^{\mathbb{Q}}\left(N_{j, t_{l}}^{\mathrm{tr}}-N_{j, t_{l-1}}^{\mathrm{tr}}\right)=E_{t}^{\mathbb{Q}}\left(N_{j, t_{l}}^{\mathrm{tr}}\right)-E_{t}^{\mathbb{Q}}\left(N_{j, t_{l-1}}^{\mathrm{tr}}\right)
$$

depend only on the portfolio loss distribution $L_{t, t_{l}}$. As shown in Section 3, this distribution can be calculated quite efficiently. In the basic AJD framework of Section 2, model-implied tranche spreads can therefore be efficiently calculated, even though they are not known in closed-form.

\section{Stochastic and Serially Correlated Recovery Rates}

This section shows that stochastic and serially correlated recovery rates can be incorporated into any tranche pricing model that allows for the decomposition (22). To this end, let $U_{k}$ be a Markov chain with state space $\{0,1,2\}$, representing a bad, neutral and good economic environment for distressed debt, respectively. Furthermore, let $U_{0} \sim$ Uniform $(0,1,2)$ and define the time-varying matrix of transition probabilities as

$$
Q_{k-1, k}^{U}=\left(\begin{array}{ccc}
1-\rho_{k} & \rho_{k} / 2 & \rho_{k} / 2 \\
\rho_{k} / 2 & 1-\rho_{k} & \rho_{k} / 2 \\
\rho_{k} / 2 & \rho_{k} / 2 & 1-\rho_{k}
\end{array}\right)
$$


with $\rho_{k} \in[0,1]$. The recovery rate of the $k$ th default in the portfolio is taken to be

$$
R^{(k)}=0.1+0.3 U_{k},
$$

so that the fractional portfolio loss due to the first $k$ defaults is

$$
L_{k}=\frac{1}{m}\left(0.1 k+0.3 V_{k}\right),
$$

with $V_{k}=\sum_{l=1}^{k} U_{l}$.

It is easy to verify that the unconditional mean and standard deviation of each recovery $R^{(k)}$ is $40 \%$ and $24.5 \%$, respectively, roughly matching the values reported by Altman and Kishore (1996). For $\rho_{k}<1 / 2$, consecutive recoveries $R^{(k-1)}$ and $R^{(k)}$ are positively correlated. The conditional loss distributions $G_{k}$ in $(22)$ can be calculated efficiently via the two dimensional Markov chain $W_{k}=\left(U_{k}, V_{k}\right)$, which has transition probabilities

$$
Q_{k-1, k}^{W}\left(U_{k}, V_{k} \mid U_{k-1}, V_{k-1}\right)=Q_{k-1, k}^{U}\left(U_{k} \mid U_{k-1}\right) \mathbf{1}_{\left\{V_{k}-V_{k-1}=U_{k}\right\}} .
$$

Hence, the distribution of $W_{k}$ can be computed in a simple recursive manner, as therefore can $G_{k}$, since

$$
G_{k}(x)=\mathbb{Q}\left(L_{k} \leq x\right)=\mathbb{Q}\left(\frac{1}{m}\left(0.1 k+0.3 V_{k}\right) \leq x\right) .
$$

Figure 1 shows the portfolio loss distribution, conditional on 25 defaults, for the case of (i) stochastic and (ii) stochastic and serially correlated recovery rates with same marginal distribution of individual recovery rates. The parameters for the latter case are $\rho_{k}=0.3$ for $1 \leq k \leq m$. We see that serial correlation of recovery rates leads to much fatter tails in the portfolio loss distribution, which is potentially good news from a modeling perspective. 


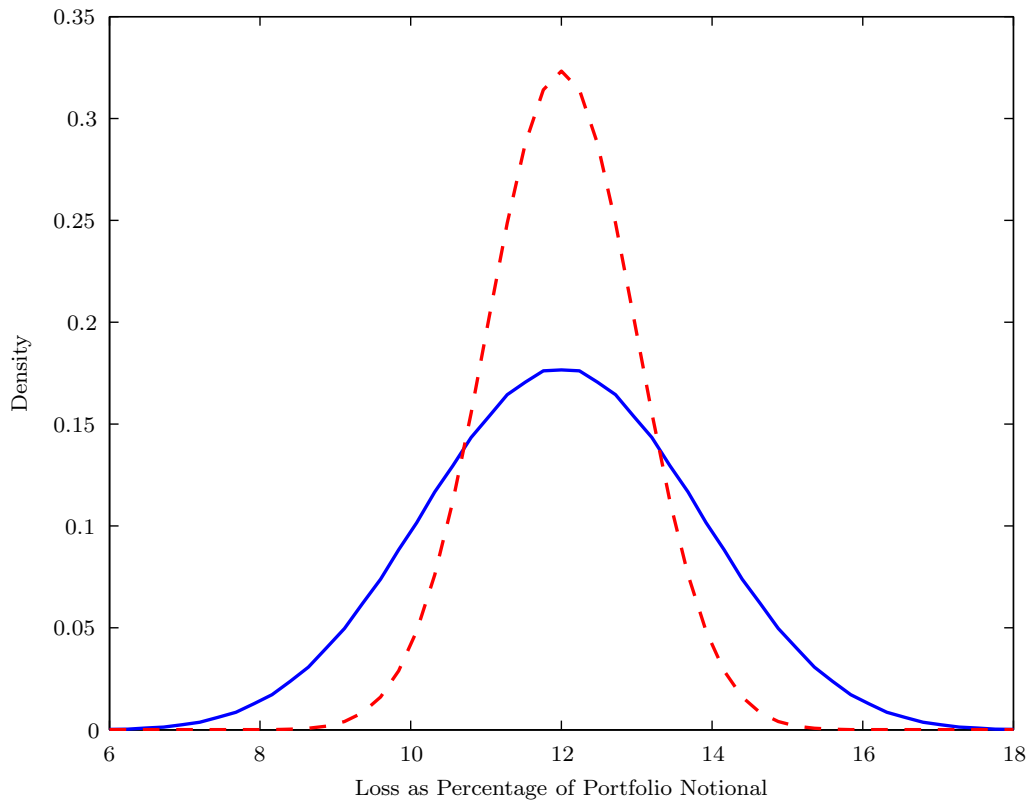

Figure 1: Portfolio loss distribution, conditional on 25 defaults, for stochastic and independent recovery rates (dashed line), and stochastic and serially correlated recovery rates (solid line) with $\rho_{k} \equiv 0.3$ in (50). 


\section{References}

Altman, E., B. Bray, A. Resti, and A. Sironi (2005). The Link between Default and Recovery Rates: Theory, Empirical Evidence and Implications. Journal of Business 78, 2203-2227.

Altman, E. I. and V. M. Kishore (1996). Almost Everything You Wanted to Know about Recoveries on Defaulted Bonds. Financial Analysts Journal 52, 57-65.

Andersen, L., J. Sidenius, and S. Basu (2003). All your hedges in one basket. Risk, $67-72$.

Berndt, A., R. Douglas, D. Duffie, M. Ferguson, and D. Schranz (2005). Measuring Default-Risk Premia from Default Swap Rates and EDFs. BIS Working Paper No. 173.

Berndt, A., R. Jarrow, and C. Kang (2007). Restructuring Risk in Credit Default Swaps: An Empirical Analysis. Stochastic Processes and Their Applications 117, 1724-1749.

Brigo, D. and A. Alfonsi (2004). Credit default swap calibration and derivatives pricing with the SSRD stochastic intensity model. Finance and Stochastics 9, 29-42.

Burtschell, X., J. Gregory, and J. Laurent (2005). A comparative analysis of CDO pricing models. Working Paper, BNP-Paribas and University of Lyon.

Carr, P. and D. B. Madan (1999). Option Valuation Using the Fast Fourier Transform. Journal of Finance 2, 61-73.

Cerny, A. (2004). Introduction to Fast Fourier Transform in Finance. Journal of Derivatives 12, 73-88.

'Credit Derivatives Handbook' (2006). Merrill Lynch.

Das, S. S., D. Duffie, N. Kapadia, and L. Saita (2007). Common Failings: How Corporate Defaults are Correlated. Journal of Finance 62, 93-117.

Delbaen, F. and W. Schachermayer (1999). A General Version of the Fundamental Theorem of Asset Pricing. Mathematische Annalen 300, 463-520.

Ding, X., K. Giesecke, and P. Tomecek (2008). Time-Changed Birth Processes and Multi-Name Credit Derivatives. Forthcoming in Operations Research.

Driessen, J. (2005). Is Default Event Risk Price in Corporate Bonds. Review of Financial Studies 18, 165-195.

Duffie, D. (1999). Credit Swap Valuation. Financial Analysts Journal 55, 73-87.

Duffie, D., A. Eckner, G. Horel, and L. Saita (2009). Frailty Correlated Default. Journal of Finance 64, 2087-2122. 
Duffie, D., D. Filipović, and W. Schachermayer (2003). Affine Processes and Applications in Finance. Annals of Applied Probability 13, 984-1053.

Duffie, D. and N. Gârleanu (2001). Risk and Valuation of Collateralized Debt Obligations. Financial Analysts Journal 57, 41-59.

Duffie, D., J. Pan, and K. Singleton (2000). Transform Analysis and Asset Pricing for Affine Jump-Diffusions. Econometrica 68, 1343-1376.

Duffie, D. and K. Singleton (1997). An Econometric Model of the Term Structure of Interest-Rate Swap Yields. Journal of Finance 52, 1287-1321.

Duffie, D. and K. Singleton (1999). Modeling Term Structures Of Defaultable Bonds. Review of Financial Studies 12, 687-720.

Duffie, D. and K. Singleton (2003). Credit Risk. Princeton, New Jersey: Princeton University Press.

Durrett, R. (2005). Probability: Theory and Examples (Third edition). Duxbury Advanced Series.

Eckner, A. (2007). Risk Premia in Structured Credit Derivatives. Working Paper, Stanford University.

Errais, E., K. Giesecke, and L. R. Goldberg (2006). Pricing Credit from the Top Down with Affine Point Processes.

Feldhütter, P. (2007). An Empirical Investigation of an Intensity-Based Model for Pricing CDO Tranches. Working paper, Copenhagen Business School.

Feldhütter, P. and D. Lando (2004). Decomposing swap spreads. EFA 2006 Zurich Meetings.

Giesecke, K. and L. R. Goldberg (2005). A Top Down Approach to Multi-Name Credit.

Harrison, M. J. and D. Kreps (1979). Martingales and Arbitrage in Multiperiod Securities Markets. Journal of Economic Theory 20, 381-408.

Harrison, M. J. and S. R. Pliska (1981). Martingales and Stochastic Integrals in the Theory of Continuous Trading. Stochastic Processes and Their Applications 11, 215-260.

Jackson, K., A. Kreinin, and X. Ma (2007). Loss Distribution Evaluation for Synthetic CDOs. Working paper, University of Toronto.

Jarrow, R. and S. Turnbull (1995). Pricing Options on Financial Securities Subject to Default Risk. Journal of Finance 50, 53-86.

Joshi, M. and A. Stacey (2006). Intensity Gamma: A New Approach to Pricing Portfolio Credit Derivatives. Working paper, Royal Bank of Scotland. 
Karatzas, I. and S. E. Shreve (2004). Brownian Motion and Stochastic Calculus (Second edition). Springer-Verlag.

Lando, D. (1998). On Cox Processess and Credit Risky Securities. Review of Derivatives Research 2, 99-120.

Li, D. (2000). On Default Correlation: A Copula Function Approach. Journal of Fixed Income 9, 43-54.

Longstaff, F. A. and A. Rajan (2008). An Empirical Analysis Of The Pricing Of Collateralized Debt Obligations. Journal of Finance 63, 529-563.

Lord, R. and C. Kahl (2006). Why the Rotation Count Algorithm Works. Tinbergen Institute Discussion Paper.

Moody's (2000). Moody's Investor Service: Historical Default Rates of Corporate Bond Issuers, 1920-1999.

Mortensen, A. (2006). Semi-Analytical Valuation of Basket Credit Derivatives in Intensity-Based Models. Journal of Derivatives 13, 8-26.

Pan, J. and K. Singleton (2008). Default and Recovery Implicit in the Term Structure of Sovereign CDS Spreads. Journal of Finance 63, 2345-2384.

Papageorgiou, E. and R. Sircar (2008). Multiscale Intensity Models and Name Grouping for Valuation of Multi-Name Credit Derivatives. Applied Mathematical Finance 15, 73105.

Protter, P. (2005). Stochastic Integration and Differential Equations (Second edition). New York: Springer-Verlag.

Schneider, P., L. Sögner, and T. Veža (2009). The Economic Role of Jumps and Recovery Rates in the Market for Corporate Default Risk. Forthcoming in Journal of Financial and Quantitative Analysis. 